\title{
Comparison of OFDMA and GFDMA for Next-Generation PONs
}

\author{
Arsalan Saljoghei, Arman Farhang, Colm Browning, Philip Perry, Nicola Marchetti, \\ Linda E. Doyle, and Liam P. Barry
}

\begin{abstract}
This paper examines and compares the use of orthogonal frequency division multiple access (OFDMA) and generalized frequency division multiple access (GFDMA) multicarrier formats in next-generation passive optical networks (PONs) through experimentation and numerical simulations. It is demonstrated that GFDMA presents a high resilience toward multiple access interference (MAI) compared to OFDMA at the uplink channel of a PON. Moreover, two methods are proposed for achieving synchronization in the uplink channel of PONs, enabling high spectral efficiency. These proposed methods can lead to the elimination of inter-ONU guard bands, and facilitate overlapping inter-ONU subcarriers, while eliminating the effects of MAI. Additionally, the performance of these multicarrier formats was analyzed in the downlink channel at a data rate of $15.6 \mathrm{~Gb} / \mathrm{s}$ and a transmission span of $50 \mathrm{~km}$ where neglible performance penalty was observed.
\end{abstract}

Index Terms-GFDMA; Multiple access; OFDMA; PON.

\section{INTRODUCTION}

$\mathbf{O}$ rthogonal frequency division multiplexing (OFDM) has been extensively explored as a potential candidate for next-generation PONs [1]. OFDM's use of multiple overlapping orthogonal subcarriers and a cyclic prefix (CP) brings about numerous benefits to PONs, such as high spectral efficiency, high resilience to chromatic dispersion, and efficent/flexible resource management [2]. These attributes are in line with the needs of next-generation PONs and have indeed been a driver behind an extensive body of work on the integration of OFDM in next-generation PONs [1]. However, OFDM suffers from a significant level of multiple access interference (MAI) in a multiuser uplink scenario. MAI arises from asynchronization of the different users' signals with respect to each other in the form of time offsets (TOs) and carrier frequency offsets (CFOs) leading to the spectral leakage problem [3]. To alleviate the effects of MAI in the uplink channel of an OFDMA system, a simple solution has been the use of adequately wide spectral guard bands, i.e., equal to the bandwidth of multiple OFDM subcarriers, between the subcarrier bands from various

Manuscript received June 13, 2017; revised September 28, 2017; accepted October 3, 2017; published November 3, 2017 (Doc. ID 298011).

A. Saljoghei (e-mail: arsalan.saljoghei@dcu.ie), C. Browning, P. Perry, and L. P. Barry are with the School of Electronic Engineering, Dublin City University, Dublin 9, Ireland.

A. Farhang, N. Marchetti, and L. E. Doyle are with CONNECT, Trinity College Dublin, Dublin, Ireland.

https://doi.org/10.1364/JOCN.9.001064 users [4]. However, this can lead to inefficient bandwidth utilization as the number of users in the network grows since the typical subcarrier bandwiths in a typcial PON could be of the order of a few megahertz. To avoid/shorten the spectral guard bands, there exists a large body of literature on the deployment of interference cancellation techniques that are computationally expensive in nature, while some of them suffer from the error propagation problem [5-8]. In a recent work $[9,10]$, the authors proposed an OFDM-based system with receiver filtering to suppress the MAI. However, this solution demands a longer CP to accommodate the receiver filter transient interval.

To tackle the aforementioned issues with OFDM, a set of new multicarrier formats has been proposed as a potential candidate for fifth-generation (5G) cellular communications. Some examples of these candidate formats are filter bank multicarrier (FBMC) and generalized frequency division multiplexing (GFDM). The two key advantages of these waveforms over OFDM are a reduction in CP demands and their capability to provide a higher resilience toward system asynchronism-reducing the potential impact of MAI. These advantages are due to the fact that these waveforms have stringent filtering properties.

In previous work [11], we analyzed and compared the performance of orthogonal frequency division multiple access (OFDMA) and FBMC multiple access (FBMC-MA) in the uplink channel of a PON using intensity modulation and direct detection (IM-DD). In that study, the effects of user asynchronism as a result of relative TOs and CFOs in the uplink channel of the PON were investigated without the incorporation of spectral inter-ONU guard bands. However, FBMC-MA-based systems exhibit a number of limiting factors compared to OFDMA, namely higher structural complexity [12] and susceptibility to sampling frequency offsets [13], along with latency and significant overheads encountered in bursty-type transmission systems [14].

In a move toward solving the inherent shortcomings of both OFDM and FBMC, waveforms such as GFDM [15] have been proposed. GFDM is an improvement to OFDM due to the fact that similar to FBMC, the filtering is imposed on each individual subcarrier to minimize the overlap between neighboring subcarriers, which can then facilitate multiuser applications in the absence of accurate synchronization of the users. The waveform design involved in GFDM also allows it to have much lower CP overheads. GFDM can be seen as an improvement over FBMC due to the potential 


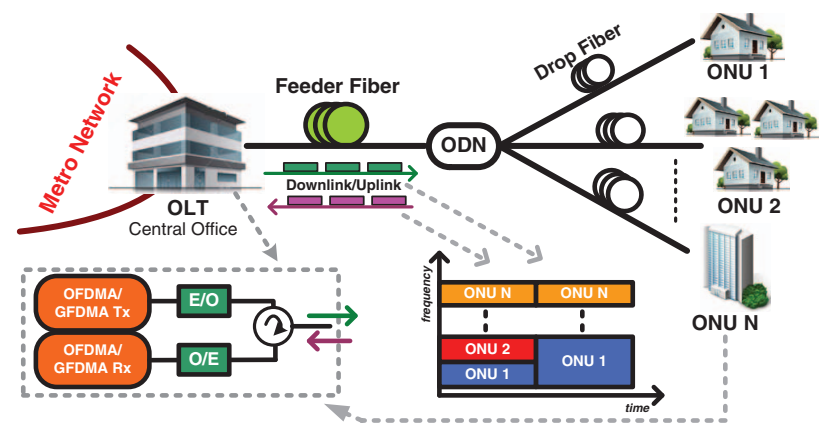

Fig. 1. Architecture of a bidirectional PON employing OFMDA/ GFDMA in both downstream and uplink.

for lower implementation complexity and lower overhead [14] in bursty-type transmission systems.

To achieve novelty in this work, we propose the use of, and also analyze the performance of, generalized frequency division multiple access (GFDMA) schemes in a bidirectional PON architecture using IM-DD, as shown in Fig. 1, and draw comparisons with OFDMA-based systems. We also avoid the employment of spectral interuser guard bands in the uplink communication channel, thereby increasing the system's overall spectral efficiency at the expense of increasing its susceptibility to MAI. In order to overcome the MAI effect in these two systems, and to eliminate asynchronism between different users, we employ the closed-loop method that we previously proposed in Ref. [11]. Additionally, to add to the novelity of this work, a simple open-loop algorithm that allows the individual users or optical network units (ONUs) to independently achieve synchronization without any management from the central station is implemented.

The rest of the paper is organized as follows. Section II begins with a review of the multicarrier formats under study along with the performance of these systems in the presence of synchronization errors. In Section III, the effect of TOs on the performance of a guard-band-less architecture at the uplink channel of PONs is analyzed through numerical simulations for the OFDMA and GFDMA formats. Section IV proposes and describes closed- and open-loop schemes that could be used for achieving synchronization in a practical PON architecture. Section V describes the experimental setup of the bidirectional PON architecture under study. Section VI presents the efficacy and performance of the uplink channel in the presence of MAI through test-bed experimentation deploying our two proposed synchronization methods. Section VII evaluates the performance of GFDM and OFDM formats at the downlink channel of the PON architecture, and Section VIII concludes the paper.

\section{WAVEFORM CHARACTERISTICS}

\section{A. OFDMA}

An OFDM signal in the time domain can be represented as

$$
x_{n, \mathrm{OFDM}}(t)=\sum_{k=0}^{N-1} s_{k, n} q\left(t-n T_{S}\right) e^{j 2 \pi k v_{o} t},
$$

where $k$ is the subcarrier index, $N$ is the total number of subcarriers, and $s_{k, n}$ is the QAM symbol modulated on subcarrier $k$ of the $n$th OFDM time symbol. $q(t)$ is an OFDM rectangular time window that is nonzero and equal to one only for $0 \leq t \leq T_{\text {OFDM }}, T_{\text {OFDM }}=T_{s}$ is the OFDM symbol duration including $\mathrm{CP}$, and $v_{0}$ is the frequency spacing between the subcarriers. It should be noted that a discrete time representation of Eq. (1) can be found in Ref. [14]. A consequence of Eq. (1) is the appearance of a sinc pulse in the frequency domain within an OFDM subband, i.e., due to the rectangular time window $q(t)$. Under ideal channel conditions, the overlapping subcarriers are orthogonal, and transmitted symbols can be recovered free of intersymbol interference (ISI) and intercarrier interference (ICI). However, in the presence of synchronization errors in the system, the orthogonality between the subcarriers breaks, which can hamper the communication. In particular, different synchronization errors of different ONUs make their subcarriers in their allocated subbands misaligned, which leads to a substantial amount of MAI [11]. This makes the integration of OFDMA in the uplink channel of multiuser networks such as PONs a challenging task if no spectral guard bands are employed or the synchronization errors are not corrected [16].

\section{B. GFDMA}

GFDM is a flexible multicarrier modulation scheme that has been utilized in Ref. [15] as an attempt to overcome the impact of MAI. The flexibility of GFDM allows it to have functionalities similar to conventional OFDM systems, single-carrier frequency-domain equalization, or singlecarrier frequency division multiplexing [17]. Moreover, like OFDM, dynamic bandwidth allocation can also be utilized in GFDMA systems. GFDM allows a CP to be shared amongst $M$ GFDM symbols. The advantage of GFDM over OFDM comes from the fact that it employs circular filtering in each subcarrier to minimize the overlap with neighboring subcarriers for facilitating multiuser applications with higher tolerance against synchronization errors. The $n$th block of a GFDM frame in time can be represented as

$$
\begin{aligned}
x_{n, \operatorname{GFDM}}(t) & =\sum_{k=0}^{N-1} \sum_{m=0}^{M} s_{k, m}^{(n)} g_{k, m}(t) q\left(t-n T_{\mathrm{GFDM}}\right) \\
g_{k, m}(t) & =g\left(\left(t-m T_{S}\right)_{\left(T_{B}\right)}\right) e^{j 2 \pi v_{o} t\left(k-\frac{N-1}{2}\right)},
\end{aligned}
$$

where $(\cdot)_{(\cdot)}$ denotes the modulo operation, and $M$ is the number of GFDM symbols overlapping with each other in one block. $T_{S}$ is the GFDM symbol duration, $T_{B}$ is the block duration, $T_{\mathrm{GFDM}}=T_{B}+T_{\mathrm{CP}}$ is the GFDM block duration including $\mathrm{CP}$, and $s_{k, m}^{(n)}$ is the QAM symbol modulated on the $m$ th symbol of subcarrier $k$ in block $n$. In Eq. (2), $g_{k, m}(t)$ has a duration of $M T_{S}$ and it is a circularly shifted version of $g_{k, 0}(t)$, which allows for pulse shaping of the transmit data symbols. It should be noted that a 
discretized realization of Eq. (2) can be found in Ref. [17]. The circular convolution allows for lower signal overheads compared with FBMC as it removes the filter transients from the beginning and end of the signal through the so-called tail biting [15]. Utilization of one CP is sufficient for one GFDM block, yielding lower CP overheads compared to OFDM with the same specifications, i.e., number of subcarriers/subcarrier spacing. The existence of a CP in GFDM compared to FBMC alleviates the need for complex receiver architectures [18] in the presence of large accumulated fiber dispersion. GFDM is categorized as a nonorthogonal waveform since unlike FBMC, it utilizes well-localized filters without the use of OQAM and this, in turn, leads to total loss of orthogonality and emergence of ICI in the system [15]. Some of the available receivers that can tackle the nonorthogonality issue in GFDM are match filtering (MF), zero forcing (ZF), and minimum mean square error (MMSE) receivers. MF receivers can maximize the signalto-noise ratio (SNR) in the system; however, they cannot remove the inherent ICI. $\mathrm{ZF}$ receivers are known to fully address the nonorthogonality problem at the expense of reducing the available SNR in the system by collecting noise from outside the desired band (see Fig. 7 in Ref. [17]). The noise enhancement property of $\mathrm{ZF}$ receivers can in turn be avoided by the deployment of MMSE receivers [17] at the expense of higher computational complexity [19]. Like FBMC [20], GFDM is known to have higher implementation complexity than OFDM. However, according to our recent work [19], the complexity of GFDM can be pushed below that of FBMC [13]. Furthermore, it has been shown in Ref. [21] that this implementation complexity can be further reduced to values close to that of OFDM.

\section{Multiple Access in UPLinK PON}

As was discussed in Ref. [11], in the uplink channel of IM-DD PONs exploiting multiple-access-type communication formats such as OFDMA and FBMC-MA without frequency guard bands, the source of desynchronization amongst ONUs could be as result of TOs and CFOs. TOs amongst ONUs originate from the fact that each ONU is connected to the optical distribution node (ODN) by a geographically independent drop fiber that can vary in length. On the other hand, the CFO effect in such architecture can originate from the potential drifts between the operational frequency of the transmit and receive local oscillators. However, the effects of CFOs can be eliminated by transmitting such multicarrier formats at the baseband as is typical in discrete multitone systems. In this work, to restrict the effects of desynchronization to TOs only, and in order to avoid the integration of complex DSP subroutines usually required to alleviate the effects of $\mathrm{CFO}$ at uplink [22], we implemented modulation at the baseband.

\section{A. Numerical Simulations}

To study the impact of TOs on the uplink channel of PONs, using the multicarrier formats described in the previous section, a number of numerical simulations are carried out in this section. In our analysis, we denote the received electrical signal from user $l$ at the OLT's uplink receiver, after the optical to electrical conversion, as $y_{l}(t)$. We assume that the received signal from a given user $l$ undergoes a TO equal to $\tau_{l}=\tau_{l \text {,feeder }}+\tau_{l \text {,drop }}$, which is attributed to the delay profile associated with both feeder and drop fibers in the link. We can write the summation of electrical signals received from all users as

$$
y(t)=\sum_{l} y_{l}\left(t-\tau_{l}\right)+n(t),
$$

where $n(t)$ is the additive white Gaussian noise (AWGN), accounting for the detector noise. After the CP removal, the OFDMA demodulation process can be represented as

$$
\hat{s}_{k, n}^{(l)}=\left\langle y(t), g_{R ; k, n}^{(l)}(t)\right\rangle=s_{k, n}^{(l)}+I_{\mathrm{MAI}}+w
$$

While for GFDMA, after discarding the $\mathrm{CP}$, it can be written as

$$
\hat{s}_{k, m, n}^{(l)}=\left\langle y(t), g_{R ; k, m}^{(l)}(t)\right\rangle=s_{k, m, n}^{(l)}+I_{\mathrm{MAI}}+I_{\mathrm{NOC}}+w
$$

where $\hat{s}$ is the detected symbol at subcarrier $k$, block $n$, and subsymbol $m$, and $g_{R ; x, y}^{(l)}(t)$ represents the analysis function or the prototype filter at the receiver shifted to $(x, y)$ frequency-time lattice points assigned to user $l$ [23]. In the above equations, the $\langle\cdot\rangle$ operator presents the projection of the received signal onto a particular analysis function at the receiver. $I_{\mathrm{MAI}}$ defines the impact of MAI on the detected symbol from neighboring subcarriers resulting from synchronization errors (a detailed analytical analysis of MAI in GFDM systems can be found in Ref. [24]). $I_{\text {NOC }}$ represents the interference obtained as a result of the nonorthogonality property of GFDM. Finally, $w$ signifies the impact of AWGN noise in the system.

In these simulations, in order to study the impact of MAI alone, we neglect the existence of noise sources and other impairments such as dispersion and sampling clock offset (SCO). However, TOs are still incorporated here, and in the case of GFDMA, $I_{\mathrm{NOC}}$ is minimized through deploying the $\mathrm{ZF}$ receiver. In our simulations, we consider two ONUs each with 32 subcarriers modulated by 16-QAM symbols. To achieve high spectral efficiency, no inter-ONU frequency guard bands were used, meaning that the highest frequency subcarrier of ONU 1 overlapped with the lowest frequency subcarrier of ONU 2. Moreover, for simplicity, we employed square root raised cosine (SRRC) prototype filters for GFDMA. Since baseband transmission is used here, the total number of available subcarriers is halved to accommodate the complex conjugate subcarrier pair for achieving Hermitian symmetry [2]. The CP can absorb the TOs and thus the MAI in both OFDMA and GFDMA can be overcome [25] as long as the TO between a target ONU and neighboring ONU is smaller than the CP size. Although this method could be useful in cellular systems [8], it cannot guarantee MAI-free transmission due to the shorter symbol periods in PON architectures [26]. 
Thus, in these simulations, we did not consider the CP for both OFDMA and GFDMA.

\section{B. Simulation Results}

The results obtained from the simulations are presented in Fig. 2. The performance of each of the 32 subcarriers used by the individual ONUs is presented in terms of error vector magnitude (EVM) with respect to the time offset $(\Delta \tau)$ between the two ONUs. The TO here is normalized to one OFDM/GFDM symbol period (i.e., $\xi=\Delta \tau / T_{s}$ ). According to Eqs. (4) and (5), since the impact of noise on the system was neglected, the increase in EVM from the baseline level as observed in the results shown in Fig. 2 can be purely attributed to the MAI effects imposed by the TOs. Comparing Figs. 2(a) and 2(b), it is clear that in the presence of TOs, OFDMA suffers most from the resultant MAI, where many of the subcarriers from both users are affected. This factor can be attributed to the orthogonality constraint, which exists amongst many subcarriers. Despite GFDM's well-localized filters [14], Fig. 2(b) shows that more than one subcarrier in each ONU is affected by MAI. The fact

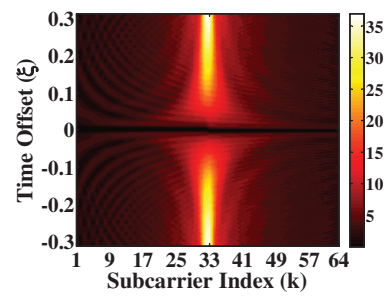

(a)

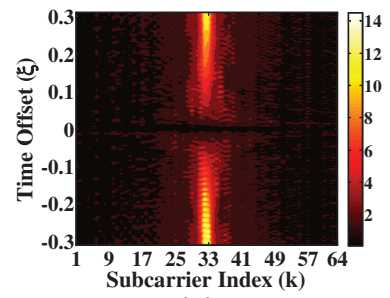

(c)

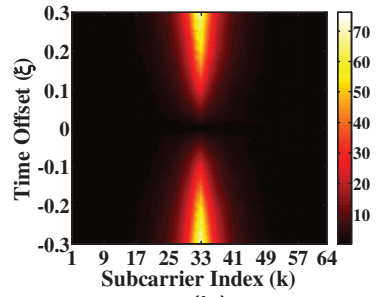

(b)

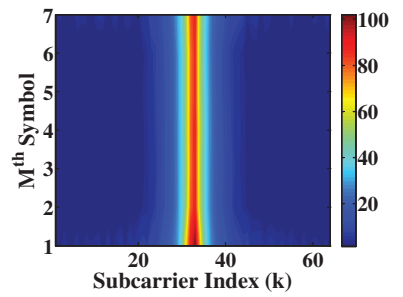

(d)

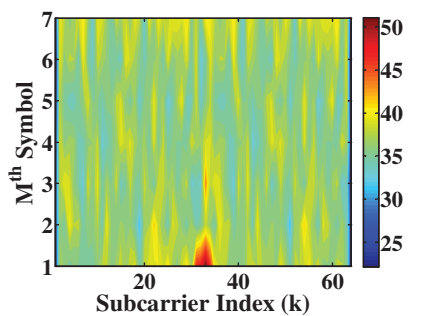

(e)

Fig. 2. Numerical simulations showing the effect of relative timing offset $(\xi)$ on the performance for each individual ONU using no frequency guard band and operating over an ideal channel free of noise and dispersion. Subcarriers 1-32: ONU 1. Subcarriers 33-64: ONU 2. (a) OFDMA, (b) GFDMA ( $\alpha=1$ ), and (c) GFDMA ( $\alpha=0.1$ ). Impact of relative timing offset set at $\xi=+0.3$ on each GFDM symbol in a block, using (d) a ZF or (e) a MF receiver with $\alpha=1$. The color bar represents the performance in EVM (\%). that more than one subcarrier is affected here, despite the use of localized filters, can be related to the high out-of-band (OOB) emission that the subcarriers in GFDM experience as a result of circular filtering. This factor is due to the presence of sharp signal transitions experienced by GFDM symbols at the beginning and end of a GFDM block [24]. Nevertheless, the high OOB emission levels can be reduced by either the deactivation of edge symbols in a GFDM block (preferably only the first symbol, as its constituent signal experiences a high level of transition) or employing windowing techniques [17]. The effect of employing techniques such as the deactivation of edge symbols or using guard subcarriers in GFDMA PONs has been studied and compared to OFDMA-PONs in our previous work [27]. Moreover, as it is shown in Fig. 2(b), the subcarriers affected in the GFDMA system for a roll-off factor of 1 exhibit a much higher degradation compared to the OFDMA in the presence of TOs, as suggested by the high EVM obtained by the inter-ONU subcarriers experiencing MAI. This can be attributed to the interaction and amplification of the $I_{\mathrm{MAI}}$ terms through the ZF receiver. However, the reduction of the filter roll-off factor leads to a significant reduction in the induced MAI and a reduction in EVM, as shown in Fig. 2(c), as result of a reduction in the overlap between neighboring subcarriers. It is well known that the reduction of the roll-off factor can lead to an increase in the number of required taps for the filtering process. However, by employing a newer class of filters such as PHYDAS as opposed to conventional filters such as SRRC filters, which also satisfy the Nyquist criterion [13], it is possible to truncate the filter's response to reduce complexity without affecting the performance.

To better understand the impact of MAI in GFDMA and its interplay with the ZF receiver, Fig. 2(d) shows the performance of each individual $M$ th GFDMA symbol as a function of subcarrier index in terms of EVM. In this figure, the roll-off factor of the prototype functions is set to 1 and the time offset is $\xi=+0.3$. As it is apparent, many of the subcarriers from each ONU are affected by MAI over all M symbols, and even a larger number of subcarriers is affected by MAI at edge symbols $(M=1,7)$ due to the higher OOB emission experienced by the subcarriers of these two edge symbols. In GFDMA, MAI should only significantly affect a small number of subcarriers at the first GFDMA symbol (as will be the case if a MF receiver is employed). However, the ZF receiver increases the leakage across many subcarriers, which give rise to the spread of MAI across all subcarriers and GFDM symbols, as shown in Fig. 2(d). This observation is due to the fact that the ZF receiver has a noise enhancement property, where it filters some out-of-band products corresponding to many of the neighboring subcarriers [17]. In the presence of desynchronization in the uplink system, the noise enhancement property of the ZF receiver of one ONU at the OLT leads to the reception and the amplification of spectral content of the neighboring ONUs, which have a time offset with respect to the primary ONU. Figure 2(e) shows the results of a similar system that was evaluated in Fig. 2(d) with the difference being that a MF receiver was used. In this figure the unaccounted $I_{\text {NOC }}$ terms lead to a high degradation across 
all symbols and subcarriers; however, as can be seen, MAI is confined mostly to the first GFDM symbols and few interONU subcarriers.

It is known that the reduction in the roll-off factors of the employed prototype filters results in the extension of the filter's impulse response $g(t)$, whose truncation in systems such as FBMC can lead to performance degradation [14]. In contrast, by comparing Fig. 2(b) with Fig. 2(c), it can be seen that GFDM shows resilience toward potential performance degradations brought about by the truncation effect. This can be attributed to the fact that the interference experienced by the truncation process contributes to the $I_{\text {NOC }}$ terms in the system, which is later discarded by the $\mathrm{ZF}$ receiver.

Lastly, it should be noted that GFDM-based systems like OFDM can also be affected by the SCO that can potentially cause MAI in uplink PON where no guard bands are used. However, in a practical system, the necessary synchronization algorithms are implemented to minimize the effect of SCO at the OLT and ONUs. In addition, it is well known that the presence of a CP in OFDM can alleviate the impact of residual SCO in the system [28]. Thankfully, GFDM also employs a CP like OFDM. Thus, the presence of a CP and an adequate synchronization subsystem for SCO can minimize the potential for degradations caused by MAI through SCO.

\section{Synchronization In Uplink PON}

Regardless of the deployed modulation format, varying degrees of MAI will always be present in the absence of the guard band due to TOs inherent to the system. Hence, efficient methods for TO correction need to be integrated into the PON architectures so that system synchronization can be achieved. Such methods will require the estimation of the individual end-to-end time delay $\left(\tau_{l}\right)$ experienced by each user. However, the challenge is that these time delays are not static, i.e., they could fluctuate due to the temperature variations over the fiber path $\left(\tau_{l}=\tau_{\text {feeder }}+\Delta \tau_{\text {feeder }}+\tau_{\text {drop }, l}+\Delta \tau_{\text {drop }, l}\right)$ [29]. It should be noted that since a common feeder is shared amongst multiple users, the delays in the drop fibers $\left(\tau_{\mathrm{drop}, l}+\Delta \tau_{\mathrm{drop}, l}\right)$ will only contribute to TOs. The time-dependent fluctuation of TOs necessitates the need for a simple system, operating at an adequate speed, capable of continuous monitoring, and correcting for the imposed time delays. These variations in TOs can be attributed to the change in the group delay along a given drop fiber as a result of temperature fluctuations $(\Delta T)$ defined in Ref. [29]. In Ref. [29], it was shown that these variations are directly proportional to the fiber length and the imposed temperature changes (assuming a uniform shift of temperature across the fiber).

\section{A. Closed-Loop Method}

A closed loop means of communication between the ONUs and the OLT is an effective way of achieving synchronization in the uplink channel of PONs [11]. This is done by obtaining an estimate for individual end to end delays between the OLT and the ONUs. This closed-loop system operates by the OLT first measuring the time delay between the received signals $y_{l}(t)$ from individual ONUs and their respective receiver time windows $\left(\tau_{\text {window, } l}\right)$ for the alignment of the receiver FFT window, which is crucial for ISI/ICI-free demodulation [28]. Figure 3(c) shows the time-domain representation of the received signals from $N$ ONUs at the OLT's receiver for an OFDMA-PON architecture. As is evident, all the users except for user 1 attain a misalignment with respect to the receiver window, which in a practical system can generally be attributed to the group delay in the fiber $\left(\tau_{l}\right)$. The determined delay values of $\zeta_{l}=\tau_{\text {window }, l}$ from each ONU can then generate insight into the time offset between various users, i.e., $\zeta_{k, p}=\tau_{\text {window }, k}-\tau_{\text {window }, p}$. To achieve synchronization, this time offset needs to be eliminated $\left(\zeta_{k, p} \approx 0\right)$. This is done by the individual ONUs delaying their uplink signals according to delays measured $\left(\zeta_{l}\right)$ by the OLT during an earlier transmission. Thanks to the periodic variations of the level of MAI experienced, which is related to the imposed TOs, which has a period equal to $T_{S}$ in OFDMA and $T_{B}$ in GFDMA (not shown in Fig. 2 due to the limited range of TOs), the delay applied to individual uplink signals do not have to be equal to the actual delay experienced over fiber (i.e., $\zeta_{l} \neq \tau_{l}$ ), but it should be sufficient to ensure $\zeta_{k, p} \approx 0$.

\section{B. Open-Loop Method}

A simple open-loop synchronization method can be deployed where the individual ONUs independently aim to achieve synchronization without the aid of the OLT. This method operates by the ONU continuously monitoring the offset between its downlink receiver window and the downlink signal sent from the OLT ( $\left.\tau_{\text {window_downlink }, l}\right)$. Based on this obtained information, the method respectively delays the uplink transmission signal from each ONU to alleviate the TO between the signal from various ONUs arriving at the OLT. This method will achieve synchronization at the uplink by ensuring $\zeta_{k, p} \approx 0$. This simple method was first proposed for $5 \mathrm{G}$ communication systems in Ref. [30]. It can be anticipated that this approach could be integrated into a practical PON architecture, despite the potential of large optical guard bands between the downlink and uplink optical transmission windows that could be in excess of $200 \mathrm{~nm}$. Compared to the closed-loop method, this scheme eliminates the overheads and latencies associated with the transmission of synchronization information from the OLT to the ONUs. The reduced latency in this method is also important for scenarios where the TO between ONUs can change rapidly due to fast temperature fluctuation over the fiber plant [11].

\section{Experimental Setup}

Figure 3 presents the experimental setup that we employed in this paper. This setup is used to emulate a 

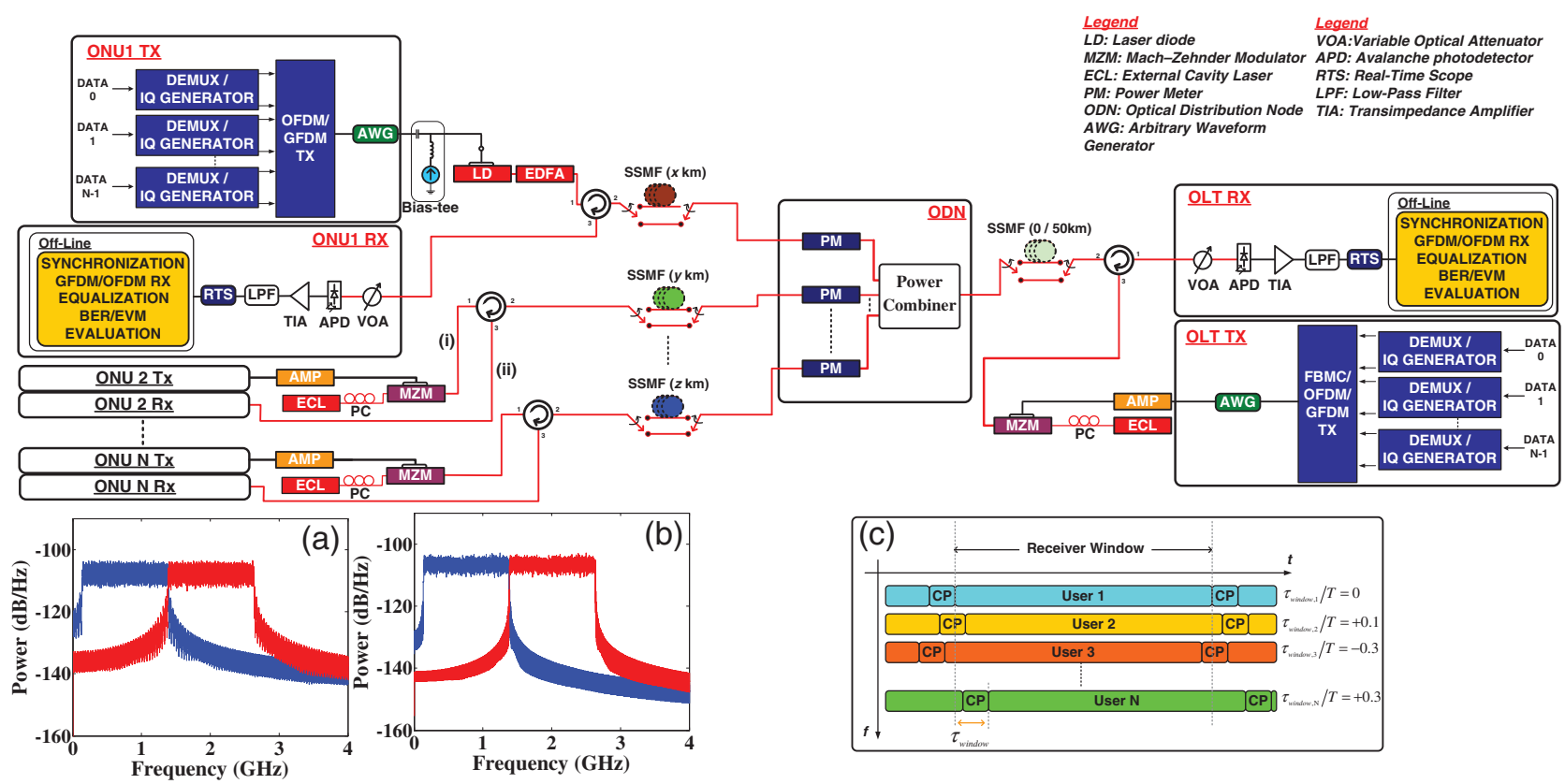

Fig. 3. Experimental setup. Electrical power spectral density transmission signal assigned to each individual ONU for (a) OFDMA-PON and (b) GFDMA-PON. (c) Time-domain representation of signals received from $N$ users at the OLT receiver of OFDMA-based PON architecture.

PON architecture operating with bidirectional traffic. For simplicity, the number of ONUs in this work was limited to two. For the uplink transmission, ONU 1 was equipped with a directly modulated single-mode laser biased at $20 \mathrm{~mA}$ and in ONU 2 an MZM biased at the quadrature point, and an external cavity laser was used. For the downlink transmission, the OLT's downlink transmitter was also equipped with an MZM. The downlink and uplink receivers of the ONUs and the OLT were fitted with an avalanche photodetector packaged with a transimpedance amplifier. Following photodetection, the analog electrical signal is digitized using a real-time scope with an integrated analog-to-digital converter operating at $10 \mathrm{GSa} / \mathrm{s}$. The digitized signals are then sent for offline processing, which entails synchronization, demodulation, channel equalization, MAI compensation, and performance evaluation in terms of EVM and bit error rate (BER).

The modulation process at the ONU's uplink transmitter and OLT's downlink transmitter is carried out offline in MATLAB and the resulting waveforms are subsequently programmed into an arbitrary waveform generator equipped with a digital-to-analog converter operating at $10 \mathrm{GSa} / \mathrm{s}$. To eliminate the need for the RF upconversion stage and restrict the impact of asynchronism to TOs only, similar to the simulation in Section III, all the waveforms were generated by using Hermitian symmetry. This approach removes the integration of a $\mathrm{RF}$ carrier for modulation and related synchronization shortcomings associated with frequency offsets. The directly modulated laser used in ONU 1 had a low output power $(<0 \mathrm{dBm})$ compared to that of ONU 2. To overcome this power difference and the potential losses between ONU 1 and the OLT, the output of this directly modulated laser was amplified using an ebrium-doped fiber amplifier (EDFA). In a practical system, it can be anticipated that each ONU will be equipped with lasers of similar output powers. Thus, there will be no need for the EDFA as used in this work. ONU 1 was assigned a drop fiber with a length of $1 \mathrm{~km}$, whereas ONU 2 was assigned a drop fiber with a length of $460 \mathrm{~m}$. The feeder span used to connect the ODN to the OLT was a $50 \mathrm{~km}$ fiber span. Furthermore, adequate input powers to the fiber links are considered for the avoidance of nonlinearities in the fiber.

Due to the employment of IM, the downlink and uplink transmitters both produce an optical double sideband with a carrier (ODSB-C) signal. To compensate for the effects of dispersive fading, which manifests itself as power loss at higher frequency subcarriers, pre-emphasis [31] is applied to both the downlink and uplink transmission links. Furthermore, to avoid possible effects of optical beat interference at the OLT's uplink receiver, the optical frequency difference between the two optical transmitters at each ONU is chosen to be greater than the bandwidth of the photodiode used at the receiver.

\section{UPLINK PERFORMANCE}

Table I summarizes the system parameters for the uplink channel, where similar parameters as the simulation studies were used for the data rate, carrier number, bandwidth assignment, and QAM order. To achieve a comparable performance between the two modulation formats, similar 
TABLE I

Uplink Physical Parameters

\begin{tabular}{lcc}
\hline Parameter & OFDMA & GFDMA \\
\hline \# users $(l)$ & 2 & 2 \\
\# available subcarriers & 128 & 128 \\
\# subcarriers per user & 32 & 32 \\
\# guard subcarriers & 0 & 0 \\
$M$ factor & 1 & 7 \\
IFFT bin size & 256 & $256 * M$ \\
CP duration & $16 / 256$ & $16 /(256 * M)$ \\
Pulse shaping $g(t)$ & - & SRRC \\
Modulation & $16-\mathrm{QAM}$ & $16-\mathrm{QAM}$ \\
Data rate per user & $5 \mathrm{~Gb} / \mathrm{s}$ & $5 \mathrm{~Gb} / \mathrm{s}$ \\
Roll-off factor $(\alpha)$ & - & 0.1 \\
Sub. spacing $\left(v_{o}\right)$ & $\sim 39 \mathrm{MHz}$ & $\sim 39 \mathrm{MHz}$ \\
Taps per subcarrier equalization & 1 & 1 \\
\# SSBI guard band & 4 & 4 \\
Training sequence overhead & $\sim 9 \%$ & $\sim 9 \%$ \\
HD-FEC overhead & $7 \%$ & $7 \%$ \\
OQAM/QAM data symbols & 29,568 & 30,000 \\
per frame per user & & \\
Total no. of frames per & 5 & 5 \\
performance evaluation & & \\
\hline
\end{tabular}

overheads were considered for channel equalization and synchronization. For error correction, a second-generation hard decision forward error correction (HD-FEC) scheme with $7 \%$ overhead was assumed [32], where the pre-FEC limit for achieving a post-FEC BER of $1 \times 10^{-15}$ was set to $2 \times 10^{-3}$.

Figures 3(a) and 3(b) depict the uplink electrical spectrum from each ONU where no frequency guard bands were used, similar to the simulations. Thus, the signals of ONU 1 and ONU 2 overlap in the frequency domain. Additionaly, as these insets show, OFDMA experiences the most significant level of inter-ONU spectral overlap, which renders it highly susceptible to MAI. GFDMA, despite using well-localized prototype filters, suffers from an extended OOB emission and leads to a great amount of spectral leakage. However, as it was discussed in Section III, this OOB emission in GFDMA leads to a lower level of MAI compared to OFDMA. Furthermore, this OOB emission in GFDMA systems is highly controllable by using filters with lower roll-off factors, windowing methods, and the deactivation of edge symbols.

The subcarrier spacing for all the waveforms was kept constant. In order to reduce the impact of the signal-tosignal beating interference (SSBI) at lower frequency bands, a guard band equal to four subcarriers was used between DC and the lowest frequency subcarrier.

The following subsections present the effect of TOs in the uplink channel of OFDMA and GFDMA PON where the effectiveness of the proposed synchronization methods for their alleviation is discussed. The experiments were carried out in a temperature-controlled laboratory environment. Thus, the fiber temperature fluctuations were slow in order to allow the operation of synchronization schemes at a slower speed, as would be expected with buried fibers.

\section{A. Closed-Loop Synchronization Scheme}

In this section, the impact of MAI induced by TOs at uplink PON and their alleviation using a closed-loop scheme is studied for the two different multicarrier schemes through experimental verification.

Figure 4(a) shows the performance of the GFDMA system in terms of BER versus received optical power with and without the closed-loop scheme for the restoration of synchronization. It is worth mentioning that no guard band in the frequency domain is considered between the ONUs. To reduce the impact of the $I_{\mathrm{NOC}}$ terms of the demodulated signal, as shown in Eq. (5), a SRRC prototype filter with the roll-off factor of 0.1 is considered [as was demonstrated in Fig. 2(c)]. As the results show, the synchronization loop successfully alleviates the time offset between users and dissipates the effects of MAI. It is clear that in the presence

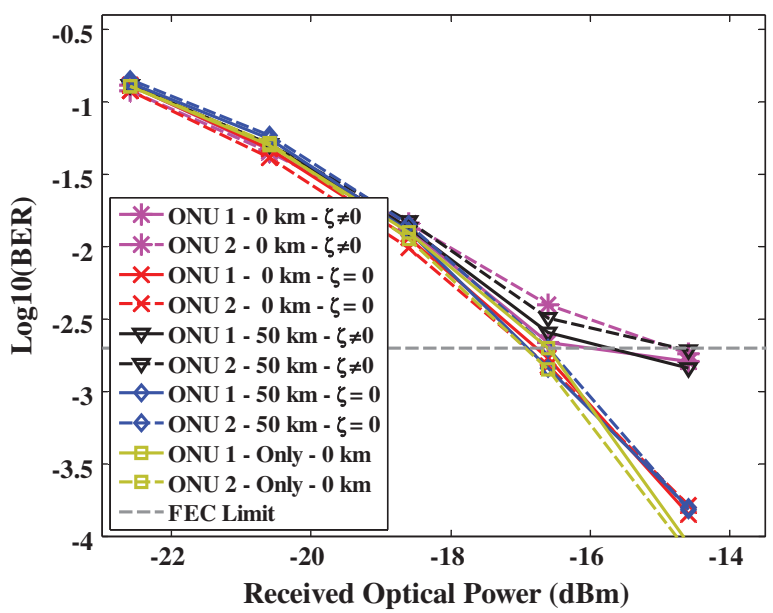

(a)

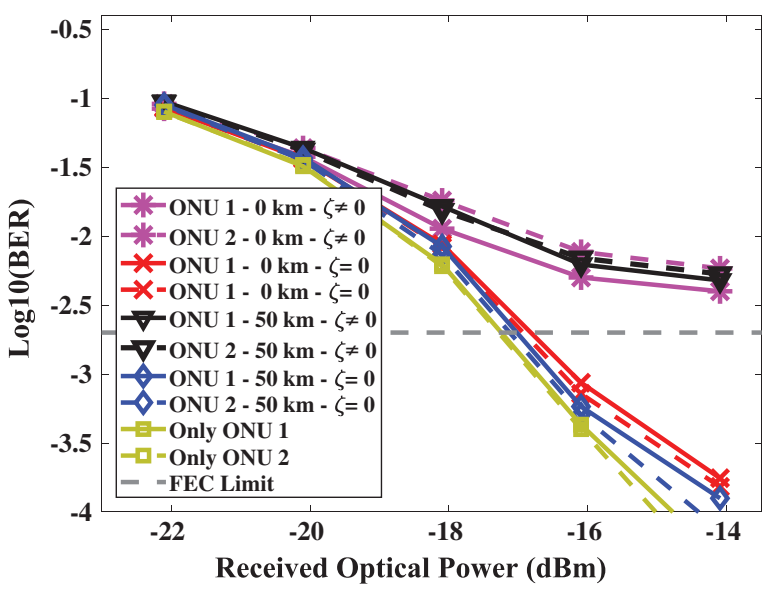

(b)

Fig. 4. Experimentally evaluated performance of two ONUs at the uplink channel in terms of BER versus received optical power in the presence/absence of a secondary ONU and feeder fiber (50 km SSMF) for (a) GFDMA and (b) OFDMA. Performance is shown before $(\zeta \neq 0)$ and after $(\zeta=0)$ the deployment of a closedloop scheme for the restoration of synchronization (ONU $\times$ Only: the modulation on the other ONU is turned off). 


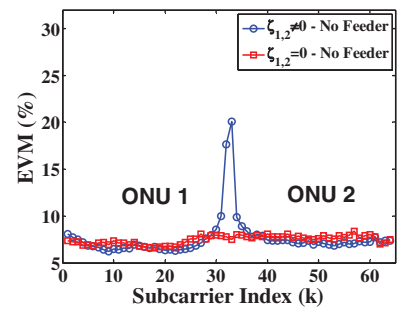

(a)

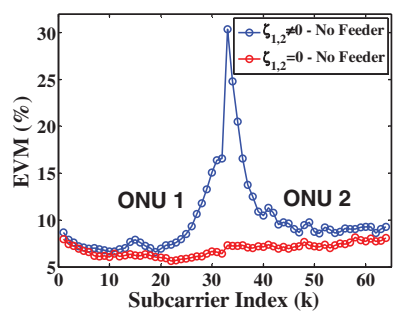

(b)
Fig. 5. Experimentally evaluated performance of two ONUs at the uplink channel in terms of EVM versus subcarrier index for (a) GFDMA and (b) OFDMA. Before $(\zeta \neq 0)$ and after $(\zeta=0)$ the deployment of a closed-loop scheme for the restoration of synchronization. (Received optical power $\approx-14 \mathrm{dBm}$.)

of TOs, GFDMA is detrimentally affected by MAI. This is evident by the attained BER floor around the FEC limit $\left(2 \times 10^{-3}\right)$ at received optical powers above $-16 \mathrm{dBm}$, which is caused by multiple inter-ONU subcarriers being subjected to MAI, as can be seen in Fig. 5(a). The trends in Fig. 5(a) show the performance of each subcarrier from individual ONUs in terms of EVM, before and after the application of closed-loop synchronization. As anticipated in the simulation results of Fig. 2(c) for $\xi \neq 0$, in the absence of synchronization, multiple inter-ONU GFDM subcarriers are affected by MAI-this factor is also seen in the experimental results of Fig. 5(a), where some subcarriers attain EVM values above $15 \%$. However, the activation of the closed-loop synchronization scheme can restore orthogonality by enforcing $\zeta=0$ and eliminating the MAI, as shown in Fig. 4(a), where the BER floor is eliminated and a BER below $1 \times 10^{-3}$ is achieved for received optical powers above $-16 \mathrm{dBm}$. Similarly, as it can be seen in Fig. 5(a), the restoration of orthogonality through the closed-loop scheme reduces the high EVM values previously seen on the inter-ONU subcarriers to that which was experienced by the unaffected subcarriers (approximately 8\%). As we had shown in our previous work [27], the impact of MAI on GFDMA can be further reduced by employing guard symbols (deactivating GFDM symbols at the edge of a block) or, as was suggested in Ref. [27], by windowing techniques. These methods can be used to overcome the high $\mathrm{OOB}$ emissions due to the edge symbols in the GFDM blocks.

Figure 4(b) presents the performance of OFDMA at uplink without any guard band. As the figure shows, in the presence of TOs and a deactivated closed-loop synchronization scheme, OFDMA suffers a significant decline in performance compared to GFDMA, leading to an error floor well above the desiered FEC limit. Thus, no performance penalty between OFDMA and GFDMA can be determined at the FEC limit. MAI affects many of the subcarriers in both ONUs, as shown in the experimental results of Fig. 5(b) and as concluded in the simulated results of Fig. 2(a). This higher degradation in turn results in a BER floor well above the FEC limit at $5 \times 10^{-3}$ for received optical powers above $-16 \mathrm{dBm}$. Nevertheless, as it is again shown in Figs. 4(a) and 5(b), the closed-loop scheme could successfully compensate for the MAI present in the system.

\section{B. Open-Loop Synchronization Scheme}

This section evaluates the efficacy and the potential for a simple open-loop scheme for achieving synchronization at the uplink.

Figure 6 presents the performance of both waveform candidates in terms of EVM versus subcarrier index for scenarios where the open-loop synchronization algorithm was either activated or not activated. As this result shows, in the absence of synchronization, EVMs of above $20 \%$ are achieved for some of the inter-ONU subcarriers as result of the MAI. However, more subcarriers are again deteriorated in the OFDMA case due to its lower resilience toward MAI as was presented previously in Figs. 4(b) and 5(b). Nevertheless, the simple open-loop scheme can successfully restore synchronization and alleviate MAI similarly to the closed-loop scheme by compensating for TOs through the enforcement of $\zeta=0$. This can be clearly observed in Fig. 6, where the application of the open-loop scheme results in all subcarriers attaining a similar EVM performance $(\sim 10 \%)$. However, it should be mentioned that despite both schemes achieving similar performance against MAI, the open-loop scheme has a much lower implementation complexity. By comparing Fig. 5 with Fig. 6 , when there is synchronization in the system, it is clear that the open-loop scheme shows performance degradation in terms of subcarrier EVM compared to the closed-loop scheme. This degradation in performance can be attributed to the fact that the results shown in Fig. 6 were evaluated at $-15 \mathrm{dBm}$ of the received optical power, which is $1 \mathrm{~dB}$ lower than the results shown in Fig. 5.

It should be noted that, although uplink performances are evaluated for two ONUs, with the implementation of an efficient timing algorithm, synchronization among a greater number of ONUs can be achieved, allowing for system expansion since successful uplink synchronization is dependent on accurate recovery of the time offset between various ONUs. Furthermore, it is clear that despite the resilience shown by GFDMA against MAI, like OFDMA, it requires a synchronization scheme to alleviate

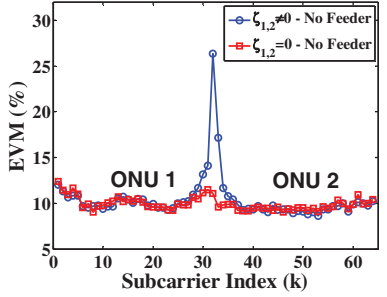

(a)

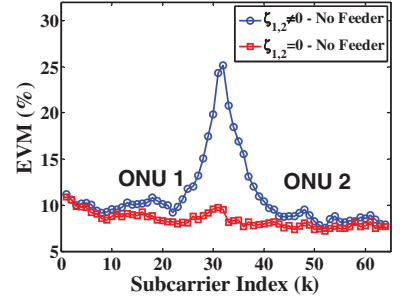

(b)
Fig. 6. Experimentally evaluated performance of two ONUs at the uplink channel using (a) GFDMA and (b) OFDMA in terms of EVM versus subcarrier index before $(\zeta \neq 0)$ and after $(\zeta=0)$ the deployment of an open-loop scheme for the restoration of synchronization. (Received optical power $\approx-15 \mathrm{dBm}$.) 
the impact of TOs. However, it should be considered that in the event of nonideal synchronization, which could be caused by rapid temperature fluctuation in the fiber plant [11], the resultant degradation caused by residual TOs is lessened in the case of GFDMA compared to OFDMA [see Figs. 2(a)-2(c)].

\section{Downlink Performance}

The performance of the downlink channel over the bidirectional fiber infrastructure is evaluated for the two different multicarrier formats operating at $15.6 \mathrm{~Gb} / \mathrm{s}$. The waveforms were generated using similar properties that were introduced in Table I, with the difference that a total of 100 subcarriers were considered for achieving the required data rates at downlink. As the results in Fig. 7 demonstrate, the GFDMA and OFDMA systems are evaluated with and without a $\mathrm{CP}$ after propagation through the feeder link. By comparing the transmission scenarios with and without a CP in Fig. 7, it is clear that these two modulation formats can achieve a CP-less transmission similar to FBMC-MA [11] with negligible deterioration to performance for the particular experimental setup shown in Fig. 3, over the designated propagation lengths and received optical power. This fact is observable by comparing the trends associated with CP-free transmission over $50 \mathrm{~km}$ of SSMF to the trends related to the cases where $\mathrm{CP}$ was adopted and to the back-to-back scenarios. As Fig. 7 suggests, all these trends converge at the FEC limit at approximately $-16.5 \mathrm{dBm}$ of received optical power. The similar levels of resilience exhibited by all modulation formats against ISI, brought upon by chromatic dispersion, are due to the signal bandwidths and data rates considered here, although operating these systems at longer propagation lengths or higher bandwidths can lead to higher $\mathrm{CP}$ overhead requirements (which will be significantly lower for GFDM) in order to ensure ISI-transmission. Nevertheless, the ODSB-C nature of intensity-modulated PONs could potentially limit the

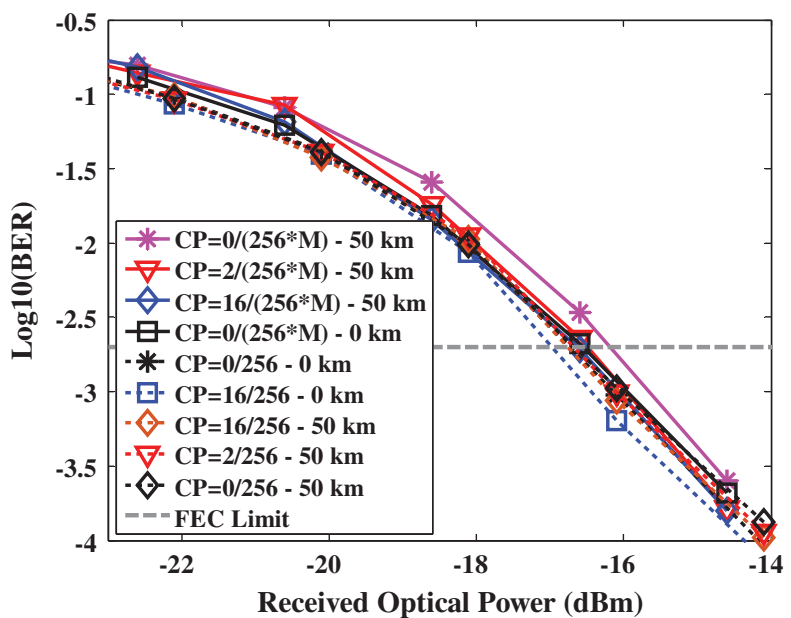

Fig. 7. Experimentally evaluated performance at the downlink channel using GFDMA (lines) and OFDMA (dashes) in terms of BER versus received optical power. performance degradations brought upon by chromatic dispersion due to the effects of dispersive fading (which manifists itself as frequency-dependent power fading) before any significant level of ISI is experienced by the various modulation formats in the type of PON architecture considered in this work.

\section{CONCLUSION}

In this work, we compared the performance of OFDMA to GFDMA in a bidirectional IM-DD PON. In order to avoid losses in the spectral efficiency at the uplink channel, no frequency guard bands were used between the spectral content of neighboring ONUs. Furthermore, the source (TOs) and resulting impact from MAI for the two modulation formats were analyzed and appropriate means for their mitigation through closed-loop and open-loop architectures were proposed and experimentally evaluated. GFDMA presented a superior performance, compared to OFDMA, against the MAI effect. This superiority results in only a limited number of inter-ONU subcarriers being affected by MAI in GFDMA which leads to BER floors below or close to the FEC limit. This is in contrast to OFDMA where BER floors well above the FEC limit were reached. However, further enhancements are possible by the integration of either windowing techniques or guard symbols.

The experimental evaluation of these modulation formats in the downlink channel of the PON system, operating at $15.6 \mathrm{~Gb} / \mathrm{s}$, has shown that in the absence of a $\mathrm{CP}$, and propagation over $50 \mathrm{~km}$ of SSMF, negligible or no degradation was observed in performance.

\section{ACKNOWLEDGMENT}

This work has been jointly supported through CONNECT (13/RC/2077) and IPIC (12/RC/2276) research grants.

\section{REFERENCES}

[1] N. Cvijetic, "OFDM for next-generation optical access networks," J. Lightwave Technol., vol. 30, pp. 384-398, 2012.

[2] J. Armstrong, "OFDM for optical communications," J. Lightwave Technol., vol. 27, pp. 189-204, 2009.

[3] C. Ruprecht, Y. Chen, D. Fritzsche, J. von Hoyningen-Huene, N. Hanik, E. Weis, D. Breuer, and W. Rosenkranz, "37.5 km urban field trial of OFDMA-PON using colorless ONUs with dynamic bandwidth allocation and TCM [Invited]," J. Opt. Commun. Netw., vol. 7, pp. A153-A161, 2015.

[4] Q. Dayou, N. Cvijetic, H. Yue-Kai, H. Junqiang, and W. Ting, "Single-wavelength $108 \mathrm{~Gb} / \mathrm{s}$ upstream OFDMA-PON transmission," in 35th European Conf. on Optical Communication (ECOC), 2009, pp. 1-2.

[5] H. Defeng and K. B. Letaief, "An interference-cancellation scheme for carrier frequency offsets correction in OFDMA systems," IEEE Trans. Commun., vol. 53, pp. 1155-1165, 2005.

[6] S. Ahmed and L. Zhang, "Low complexity iterative detection for OFDMA uplink with frequency offsets," IEEE Trans. Wireless Commun., vol. 8, pp. 1199-1205, 2009. 
[7] K. Raghunath and A. Chockalingam, "SIR analysis and interference cancellation in uplink OFDMA with large carrier frequency and timing offsets," in IEEE Wireless Communications and Networking Conf., 2007, pp. 996-1001.

[8] M. Morelli, C. C. J. Kuo, and M. O. Pun, "Synchronization techniques for orthogonal frequency division multiple access (OFDMA): A tutorial review," Proc. IEEE, vol. 95, pp. 1394 1427, 2007.

[9] A. Farhang, A. J. Majid, N. Marchetti, L. E. Doyle, and B. Farhang-Boroujeny, "Interference localization for uplink OFDMA systems in presence of CFOs," in IEEE Wireless Communications and Networking Conf. (WCNC), 2014, pp. 1030-1035.

[10] A. Farhang, N. Marchetti, L. E. Doyle, and B. FarhangBoroujeny, "Low complexity CFO compensation in uplink OFDMA systems with receiver windowing," IEEE Trans. Signal Process., vol. 63, pp. 2546-2558, 2015.

[11] A. Saljoghei, F. Gutierrez, P. Perry, D. Venkitesh, R. Koipillai, and L. Barry, "Experimental comparison of FBMC and OFDM for multiple access uplink PON," J. Lightwave Technol., vol. 35, pp. 1595-1604, 2017.

[12] A. Saljoghei, F. A. Gutiérrez, P. Perry, and L. P. Barry, "Filter bank multicarrier (FBMC) for long-reach intensity modulated optical access networks," Opt. Commun., vol. 389, pp. 110-117, Apr. 2017.

[13] B. Farhang-Boroujeny, "Filter bank multicarrier modulation: A waveform candidate for 5G and beyond," Adv. Electr. Eng., vol. 2014, 482805, 2014.

[14] B. Farhang-Boroujeny and H. Moradi, "OFDM inspired waveforms for 5G," IEEE Commun. Surv. Tutorials, vol. 18, pp. 2474-2492, 2016.

[15] G. Fettweis, M. Krondorf, and S. Bittner, "GFDMGeneralized frequency division multiplexing," in IEEE 69th Vehicular Technology Conf. (VTC), 2009, pp. 1-4.

[16] J. Zhang, X. Yi, Q. Yang, H. Zhang, M. Deng, and K. Qiu, "Collective reception of orthogonal band multiplexed data streams for OFDM-PON upstream transmissions," Opt. Commun., vol. 285, pp. 3597-3603, 2012.

[17] N. Michailow, M. Matthé, I. S. Gaspar, A. N. Caldevilla, L. L. Mendes, A. Festag, and G. Fettweis, "Generalized frequency division multiplexing for 5th generation cellular networks," IEEE Trans. Commun., vol. 62, pp. 3045-3061, 2014.

[18] J. Zhao and P. D. Townsend, "Dispersion tolerance enhancement using an improved offset-QAM OFDM scheme," Opt. Express, vol. 23, pp. 17638-17652, June 2015.

[19] A. Farhang, N. Marchetti, and L. E. Doyle, "Low-complexity modem design for GFDM," IEEE Trans. Signal Process., vol. 64, pp. 1507-1518, 2016.
[20] L. G. Baltar, F. Schaich, M. Renfors, and J. A. Nossek, "Computational complexity analysis of advanced physical layers based on multicarrier modulation," in Future Network \& Mobile Summit (FutureNetw.), 2011, pp. 1-8.

[21] P. Wei, X. G. Xia, Y. Xiao, and S. Li, "Low-complexity DGTbased GFDM receivers in broadband channels," in IEEE Int. Conf. on Communication Systems (ICCS), 2016, pp. 1-6.

[22] H. Saeedi-Sourck, Y. Wu, J. W. M. Bergmans, S. Sadri, and B. Farhang-Boroujeny, "Sensitivity analysis of offset QAM multicarrier systems to residual carrier frequency and timing offsets," Signal Process., vol. 91, pp. 1604-1612, 2011.

[23] A. Sahin, I. Guvenc, and H. Arslan, "A survey on multicarrier communications: Prototype filters, lattice structures, and implementation aspects," IEEE Commun. Surv. Tutorials, vol. 16, pp. 1312-1338, 2014.

[24] A. RezazadehReyhani and B. Farhang-Boroujeny, "Asynchronous performance of circularly pulse-shaped waveforms for 5G," arXiv:1511.07910, 2015.

[25] M. Matthé, L. L. Mendes, and G. Fettweis, "Asynchronous multi-user uplink transmission with generalized frequency division multiplexing," in IEEE Int. Conf. on Communication Workshop (ICCW), 2015, pp. 2269-2275.

[26] J. Sun-Young, S.-M. Jung, H.-J. Park, and S.-K. Han, "Mitigation of timing offset effect in IM/DD based OFDMAPON uplink multiple access," Opt. Express, vol. 23, pp. 13889-13898, June 2015.

[27] A. Saljoghei, A. Farhang, C. Browning, N. Marchetti, L. Doyle, and L. Barry, "Investigation of the performance of GFDMA and OFDMA for spectrally efficient broadband PONs," in Optical Fiber Communication Conf., Los Angeles, California, 2017, paper Th2A.25.

[28] M. Speth, S. A. Fechtel, G. Fock, and H. Meyr, "Optimum receiver design for wireless broad-band systems using OFDM. I," IEEE Trans. Commun., vol. 47, pp. 1668-1677, 1999.

[29] A. Ben-Amram, Y. Stern, Y. London, Y. Antman, and A. Zadok, "Stable closed-loop fiber-optic delay of arbitrary radio-frequency waveforms," Opt. Express, vol. 23, pp. 28244-28257, Feb. 2015.

[30] T. Wild, F. Schaich, and Y. Chen, "5G air interface design based on Universal Filtered (UF-)OFDM," in 19th Int. Conf. on Digital Signal Processing, 2014, pp. 699-704.

[31] Y. Gao, J. Yu, J. Xiao, Z. Cao, F. Li, and L. Chen, "Directdetection optical OFDM transmission system with preemphasis technique," J. Lightwave Technol., vol. 29, pp. 2138-2145, 2011.

[32] "Forward error correction for high bit-rate DWDM submarine systems," ITU-T Recommendation G.975.1, Feb. 2004. 